\title{
NEW EVIDENCE FOR NON-COSMOLOGICAL REDSHIFT
}

\author{
YAOQUAN CHU AND XINGFEN ZHU \\ Center for Astrophysics, Univ. of Sci. and Tech. of China \\ Hefei, Anhui 230026, China
}

Recently Arp (1997) has published a list of x-ray emitting blue stellar objects (BSO) around 24 Seyfert galaxies. Herewith we present our optical identification of 5 BSOs in the field NGC3516 obtained on April 4-5, 1997 using the $2.16 \mathrm{~m}$ telescope at Xinlong Station, Beijing Astronomical Observatory. One of the objects Q1107+7232 with $\mathrm{z}=2.10\left(\theta=4.34^{\prime}, \theta\right.$ is the angular distance from the center of NGC3516) is already listed in the Hewitt-Burbidge Catalog (1993). We find the other four objects are all quasars: Q1108+7226 with $\mathrm{z}=0.328\left(\theta=11.23^{\prime}\right) ; \mathrm{Q} 1106+7244, \mathrm{z}=0.690$ $\left(\theta=10.23^{\prime}\right) ; \mathrm{Q} 1105+7242, \mathrm{z}=0.930,\left(\theta=10.99^{\prime}\right) ; \mathrm{Q} 1105+7238, \mathrm{z}=1.399$ $\left(\theta=7.42^{\prime}\right) ; \mathrm{Q} 1107+7232, \mathrm{z}=2.10\left(\theta=4.34^{\prime}\right)$.

We find these quasars show a very good correlation between their redshifts and angular distances from NGC3516.The statistical analyses show that the linear regression is $z=3.06-0.22 \theta$, with the correlation coefficient $=-0.957$ and the standard error of the regression line $(\mathrm{Sy} / \mathrm{x})=0.23$. If these quasars were ejected from central galaxy, it means that the younger the quasar, the closer it is to the center, and the higher its redshift. These 5 quasars located less than 12 arc min from the X-ray Seyfert galaxy NGC3516 and distributed along the minor axis of this galaxy within $+/-20$ degree. Moreover, the 5 quasars just have redshift values which very close to the peaks of redshift periodicity: $\mathrm{z}=0.3,0.6,0.96,1.41,1.96$, which is consistent with the result that multi-quasars are associated with low redshift galaxies(Zhu and Chu, 1990). All of the interesting features indicated above for these 5 quasars in the NGC3516 field are not easily interpreted using a cosmological origin of quasar redshift. We conclude that this is a further new evidence that quasars have significant non-cosmological redshift components.

\section{References}

Arp. H., (1997), $A A$ p.,319, p.33.

Zhu,X.and Chu, Y., (1990), Acta Astronomica Sinica, 31,p.132. 